\title{
ON ONE TYPE \\ OF COMPACTIFICATION OF POSITIVE INTEGERS
}

\author{
MiLAN PAŠTÉKA
}

\begin{abstract}
The object of observation is a compact metric ring containing positive integers as dense subset. It is proved that this ring is isomorphic with a ring of reminder classes of polyadic integers.
\end{abstract}

Let $\mathbb{N}$ be the set of positive integers. A mapping $\|\cdot\|: \mathbb{N} \rightarrow<0, \infty)$ will be called norm if and only if the following conditions are satisfied for $a, b \in \mathbb{N}$

$$
\|a\|=0 \Leftrightarrow a=0, \quad\|a+b\| \leq\|a\|+\|b\|, \quad\|a b\| \leq\|b\| .
$$

There are various examples of norms on $\mathbb{N}$. One of these is polyadic norm defined in $[\mathrm{N}]$, [N1]. We start by a generalization of polyadic norm. Denote by $a+(m)$ the arithmetic progression with difference $m$ which contains $a$. Instead of $0+(m)$ we write only $(m)$.

A subset $A \subset \mathbb{N}$ we call closed to divisibility or shortly CD-set if and only if

$$
1 \in A, \quad m \in A, d \mid m \Rightarrow d \in A, \quad m_{1}, m_{2} \in A \Rightarrow\left[m_{1}, m_{2}\right] \in A,
$$

for $d, m, m_{1}, m_{2} \in \mathbb{N}$.

Suppose that $A$ is infinite $C D$-set and $\left\{B_{n}\right\}$ is such sequence elements of $A$ that for every $d \in A$ there exists $n_{0}$ that $d \mid B_{n}$ for $n>n_{0}$. It is easy to see that the mapping

$$
\|a\|_{A}=\sum_{n=1}^{\infty} \frac{h_{n}(a)}{2^{n}}
$$

for $a \in \mathbb{N}$, where $h_{n}(a)=1-\mathcal{X}_{\left(B_{n}\right)}$, is a norm. This norm will be called generalized polyadic norm and the completion with respect the metric given by this norm will be called the ring of generalized polyadic integers.

(C) 2015 Mathematical Institute, Slovak Academy of Sciences. 2010 Mathematics Subject Classification: 11 K06.

Keywords: density, polyadic integers.

Research was supported by grant VEGA 2/0146/14. 


\section{MILAN PAŠTÉKA}

If $A=\mathbb{N}$ and $B_{n}=n$ !, we get polyadic norm and the completion will be the ring of polyadic integers. In the case $A=\left\{p^{n} ; n=0, ., 2, ..\right\}$ and $B_{n}=p^{n}$ for a given prime $p$ we a obtain $p$-adic norm and the completion will be the ring of $p$-adic integers.

In the following text we shall assume that there is given a compact metric space $(\Omega, \rho)$ containing $\mathbb{N}$ as a dense subset. We suppose that the operations addition and multiplication on $\mathbb{N}$ are continuous and are extended to whole $\Omega$ to continuous operations. Thus $(\Omega,+, \cdot)$ is a topological commutative semiring.

Since $\Omega$ is compact, we can suppose that there exists an increasing sequence of positive integers $\left\{x_{n}\right\}$ convergent to an element of $\Omega$. Put

$$
a_{n}=x_{2 n}-x_{n}, \quad n=1,2, \ldots
$$

Then

$$
a_{n} \geq n \quad \text { and } \quad a_{n} \rightarrow 0
$$

in the topology of $\Omega$.

For $\beta \in \Omega$ and $b_{n} \rightarrow \beta, b_{n} \in \mathbb{N}$ we can consider the sequence of positive integer $\left\{a_{k_{n}}-b_{n}\right\}$, for a suitable increasing sequence $\left\{k_{n}\right\}$, such that

$$
a_{k_{n}}-b_{n} \rightarrow \beta^{\prime}, \quad \text { where } \beta+\beta^{\prime}=0 .
$$

We see that $(\Omega,+)$ is a compact group.

Clearly, for every $m \in \mathbb{N}$ there holds $c l(r+(m))=r+m \Omega$, where $m \Omega$ is the principal ideal in the ring $(\Omega,+, \cdot)$ generated by $m$. This yields

$$
\Omega=m \Omega \cup(1+m \Omega) \cup \cdots \cup(m-1+m \Omega) .
$$

Since the divisibility by $m$ in $\mathbb{N}$ is not necessary equivalent with the divisibility by $m$ in $\Omega$, it is not assumed that the last decomposition is disjoint.

LEMma 1. Let $m \in \mathbb{N}$ be such positive integer that it is also the minimal generator of the ideal $m \Omega$. Then every positive integer is divisible by $m$ in $\mathbb{N}$ if and only if it is divisible by $m$ in $\Omega$.

P r o of. One implication is trivial. Suppose now that some positive integer $a$ is divisible by $m$ in $\Omega$. Thus $a \in m \Omega$. Put $d=(a, m)$ - the greatest common divisor in $\mathbb{N}$. Then $d=a x+m y$ for certain integers $x, y$. This yields $d \in m \Omega$. We get $d \Omega=m \Omega$ and the minimality of $m$ implies $m=d$.

For every $n \in \mathbb{N}$ we can define $g(n)$ as the minimal positive generator of $n \Omega$. Put $\mathcal{A}=\{g(n) ; n \in \mathbb{N}\}$.

The set $r+m \Omega$ is closed and so from (2) we see that also open, which we refer as clopen set.

It is easy to check that the set $\mathcal{A}$ is a CD-set. 
Let $\left\{a_{n}\right\}$ be the sequence of positive integers given in (11). Clearly,

this yields

$$
\bigcap_{n=1}^{\infty} a_{n} \Omega=\{0\}
$$

$$
\bigcap_{m \in \mathcal{A}} m \Omega=\{0\} .
$$

So we obtain that the set $\mathcal{A}$ is infinite. Since $m \Omega$ is open for $m \in \mathcal{A}$, equality (3) implies that for each sequence $\left\{\alpha_{n}\right\}$ there holds

$$
\alpha_{n} \rightarrow 0 \Longleftrightarrow \forall m \in \mathcal{A} \exists n_{0} ; \quad n \geq n_{0} \Longrightarrow m \mid \alpha_{n} .
$$

If we define the congruence by the natural manner: $\alpha \equiv \beta(\bmod \gamma)$ if and only if $\gamma$ divides $\alpha-\beta$, for $\alpha, \beta, \gamma \in \Omega$, then there holds:

$$
\alpha_{n} \rightarrow \beta \Longleftrightarrow \forall m \in \mathcal{A} \exists n_{0} ; \quad n \geq n_{0} \Longrightarrow \alpha_{n} \equiv \beta \quad(\bmod m) .
$$

Thus the convergence can be metrised by the generalized polyadic norm. Let

$$
\mathcal{A}=\left\{m_{n}, n=1,2, \ldots\right\} \quad \text { and } \quad M_{n}=\left[m_{1}, \ldots, m_{n}\right], n=1,2, \ldots,
$$

then

We get the following

$$
\|\alpha\|_{\mathcal{A}}=\sum_{n=1}^{\infty} \frac{1-\mathcal{X}_{M_{n} \Omega}(\alpha)}{2^{-n}}
$$

Theorem 1. The metric $\rho$ is equivalent with the metric $\rho_{\mathcal{A}}$, where

$$
\rho_{\mathcal{A}}(\alpha, \beta)=\|\alpha-\beta\|_{\mathcal{A}} \quad \text { for } \alpha, \beta \in \Omega \text {. }
$$

So for every set $S \subset \Omega$ we have

$$
c l(S)=\bigcap_{n=1}^{\infty}\left(S+M_{n} \Omega\right) .
$$

Denote the Haar probability measure defined on $(\Omega,+)$ by $P$. For $m \in \mathcal{A}$ the decomposition (2) is disjoint and so $P(r+m \Omega)=\frac{1}{m}$. If we define the submeasure $\nu^{*}$ on the system of subsets of $\mathbb{N}$ as $\nu^{*}(S)=P(\operatorname{cl}(S))$, we get from (4) and upper semicontinuity of measure that for each $S$

$$
\nu^{*}(S)=\lim _{n \rightarrow \infty} \frac{R\left(S: M_{n}\right)}{M_{n}},
$$

where $R\left(S: M_{n}\right)$ the number of elements of $S$ incongruent modulo $M_{n}$. Thus $\nu^{*}$ is the covering density defined in $[\mathrm{P}]$.

TheOREM 2. Let $\alpha, \beta \in \Omega$. There exist $\alpha_{1}, \beta_{1} \in \Omega$ such that the element $\delta=\alpha_{1} \alpha+\beta_{1} \beta$ divides $\alpha$ and $\beta$. 
Proof. Let $\left\{a_{n}\right\},\left\{b_{n}\right\}$ be the sequences of positive integers that $a_{n} \rightarrow$ $\rightarrow \alpha, b_{n} \rightarrow \beta$. Let $d_{n}$ the greatest common divisor of $a_{n}, b_{n}, n=1,2, \ldots$ Then $d_{n}=v_{n} a_{n}+u_{n} b_{n}$ for some $u_{n}, v_{n}$-integers. The compactness of $\Omega$ provides that $u_{k_{n}} \rightarrow \alpha_{1}$ and $v_{k_{n}} \rightarrow \beta_{1}$ for a suitable increasing sequence $\left\{k_{n}\right\}$. Put $\delta=\alpha_{1} \alpha+\beta_{1} \beta$. We see that $d_{k_{n}} \rightarrow \delta$. For $n=1,2, \ldots$ we have $a_{k_{n}}=c_{n} d_{k_{n}}$. Since $\left\{c_{n}\right\}$ contains a convergent subsequence, we get that $\delta$ divides $\alpha$. Analogously, it can be derived that $\delta$ divides $\beta$.

The element $\delta$ from Theorem 2 will be called the greatest common divisor of $\alpha, \beta$ and we shall write $\delta \sim(\alpha, \beta)$.

Corollary 1. If $p \in \mathcal{A}$ is a prime then for every $\alpha \in \Omega$ there holds $p$ divides $\alpha$ or $(\alpha, p) \sim 1$.

P r o o f. If $p$ does not divide $\alpha$ then $\alpha \in \Omega \backslash p \Omega$. Consider a sequence of positive integers $\left\{a_{n}\right\}$ which converges to $\alpha$. The set $\Omega \backslash p \Omega$ is open, thus we can suppose that $\left(a_{n}, p\right)=1$. This yields $\ell_{n} a_{n}+s_{n} p=1$ for suitable integers $\ell_{n}, s_{n}$. Since $\Omega$ is a compact space there exists an increasing sequence $\left\{k_{n}\right\}$ that $\ell_{k_{n}} \rightarrow \lambda, s_{k_{n}} \rightarrow \sigma$. And so $\lambda \alpha+\sigma p=1$.

Corollary 2 can be proved analogously

Corollary 2. An element $\alpha \in \Omega$ is invertible if and only if $(\alpha, p) \sim 1$ for every prime $p \in \mathcal{A}$.

Lemma 2. Each closed ideal in $\Omega$ is principal ideal.

Proof. Let $I \subset \Omega$ be closed ideal. Let $\alpha \in I$. Denote by $I_{\alpha}$ the set of all divisors of $\alpha$ belonging to $I$. The compactness of $\Omega$ yields that $I_{\alpha}$ is a closed set. From Lemma 2 we get that for every $\alpha, \beta \in I$ there exists $\delta \in I$ so that $I_{\delta} \subset I_{\alpha} \cap I_{\beta}$. And so it can be proved by induction that $I_{\alpha}, \alpha \in I$ is a centered system of closed sets. Thus its intersection is non empty, and contains an element $\gamma$. Then $I=\gamma \Omega$.

In the sequel we denote $\Omega$ the ring of polyadic integers, thus completion of $\mathbb{N}$ with respect to norm $\|\cdot\|_{\mathbb{N}}$ and we suppose that an infinite CD-set $A$ is given. The completion of $\mathbb{N}$ with respect to the norm $\|\cdot\|_{A}$ we denote as $\Omega_{A}$.

Lemma 2 provides that $\cap_{a \in A} a \Omega=\alpha \Omega$ for suitable $\alpha \in \Omega$.

We will prove the folowing

TheORem 3. The ring $\Omega_{A}$ is isomorphic with the factor ring $\Omega / \alpha \Omega$.

Proof. If a sequence of positive integers is Cauchy's with respect to $\|\cdot\|_{\mathbb{N}}$, then it is Cauchy's with respect to $\|\cdot\|_{A}$ as well.

If $\left\{a_{n}\right\},\left\{b_{n}\right\}$ are sequences of positive integer, then

$$
||\left|a_{n}-b_{n}\right|||_{\mathbb{N}} \rightarrow 0 \Longrightarrow||\left|a_{n}-b_{n}\right|||_{A} \rightarrow 0 .
$$




\section{ON ONE TYPE OF COMPACTIFICATION OF POSITIVE INTEGERS}

Therefore we can define a mapping $F: \Omega \rightarrow \Omega_{A}$ in the following way. If $\beta \in \Omega, b_{n} \rightarrow \beta$ with respect to $\|\cdot\|_{\mathbb{N}}$, then $F(\beta)$ is the limit of $\left\{b_{n}\right\}$ with respect to $\|\cdot\|_{A}$. Clearly, $F$ is a surjective morphism with kernel $\cap_{a \in A} a \Omega$ and the assertion follows.

TheOREM 4. The ring $\Omega_{A}$ is an integrity domain if and only if $A=\left\{p^{n} ; n=\right.$ $0,1,2 \ldots\}$, where $p$ is a prime number.

P r o of. Suppose that $A=\left\{p^{n} ; n=0,1,2 \ldots\right\}$. It suffices to prove that $\cap_{a \in A} a \Omega$ is a prime ideal. Let $\alpha, \beta$ do not belong to $\cap_{a \in A} a \Omega$. Then $\alpha=p^{k} \alpha_{1}, \beta=p^{j} \beta_{1}$, where $\left(p, \alpha_{1}\right) \sim 1,\left(p, \beta_{1}\right) \sim 1, j, k<\infty$. Thus

$$
\alpha \beta=p^{k+j} \alpha_{1} \beta_{1} \notin \cap_{a \in A} a \Omega .
$$

Assume that $A$ contains at least two different primes. The elements of the sequence $\left\{M_{k}\right\}$ can be decomposed into $M_{k}=d_{k} c_{k}$ such that

$$
\left(d_{k}, c_{k}\right)=1, \quad d_{k}>1, \quad c_{k}>1, \quad k=1,2, \ldots
$$

Let $\left\{k_{n}\right\}$ be a subsequence such that $d_{n_{k}} \rightarrow \delta, c_{n_{k}} \rightarrow \gamma$. Then $\gamma, \delta \notin \cap_{a \in A} a \Omega$ and $\gamma \delta \in \cap_{a \in A} a \Omega$, thus $\cap_{a \in A} a \Omega$ is not a prime ideal.

\section{REFERENCES}

[N] NOVOSELOV, E. V.: Topological theory of polyadic numbers, Trudy Tbilis. Mat. Inst. 27 (1960), 61-69. (In Russian)

[N1] NOVOSELOV, E. V.: New method in the probability number theory, Doklady Akademii Nauk. Ser. Mat. 28 (1964) no. 2, 307-364. (In Russian)

[P] PAŠTÉKA, M.: Covering densities, Math. Slovaca 42 (1992), 593-614.

[Po] POSTNIKOV, R.G.: Introduction to Analytic Number Theory. Moscov, Nauka, 1971. (In Russian); Amer. Math. Soc., Providence RI, 1981 (English translation).

[P-P] PAŠTÉKA, M.-PORUBSKÝ, Š.: On the distribution on the sequences of integers, Math. Slovaca 43 (1993), 521-639.

\author{
Department of Mathematics and \\ Computer Science \\ Faculty of Education \\ University of Trnava \\ Priemyselná 4 \\ SK-918-43 Trnava \\ SLOVAKIA
}

E-mail: milan.pasteka@mat.savba.sk 